À propos de...

Gilles Laferté, L'embourgeoisement : une enquête sur les céréaliers, Paris, Raisons d'agir (Cours et travaux), 2018, $376 \mathrm{p}$.

\title{
Ni tout à fait paysan, ni tout à fait bourgeois
}

\author{
Camille Herlin-Giret
}

L'ouvrage de Gilles Laferté s'inscrit dans un cheminement méthodologique et conceptuel. Méthodologique d'abord, car l'ethnographie proposée est originale à deux égards. Elle porte sur un espace rural, le Germanois, qui fait l'objet d'une démarche collective d'enquête de longue durée ${ }^{1}$. Au-delà de la mutualisation des données, qui vient enrichir la recherche, cette démarche ouvre la possibilité à l'auteur de multiplier les points de vue sur un objet et de réaliser davantage de connexions entre un monde - celui des agriculteur'rices, au centre de l'ouvrage - et d'autres - les ouvrier·es du Germanois par exemple. L'approche est aussi socio-historique. À l'appui d'archives municipales et départementales et d'un travail sur le recensement - des matériaux mis en regard des entretiens conduits-, l'ouvrage suit plusieurs générations d'agriculteur rices et retrace plus largement les relations entre différents groupes d'interconnaissance.

L'ethnographie historique d'un groupe localisé peut rapidement tomber dans l'écueil monographique. Une des forces du livre de Gilles Laferté est bien de mettre la richesse des matériaux au service d'une réflexion conceptuelle qui poursuit le projet de « dissoudre la sociologie rurale et l'ethnologie de la France dans une sociologie sinon des classes, a minima, de la stratification sociale ${ }^{2} »$. Plus qu'un groupe ou un espace, c'est d'ailleurs un processus, l'embourgeoisement, qui est au cœur du livre. Pour comprendre ce mouvement long, l'approche proposée se veut relationnelle³. L'enjeu est, d'une part, de ne pas réduire l'étude d'un groupe à une dimension - travail, logement, loisirs, rapport au politique, etc. - pour prendre en compte l'ensemble des relations et des pratiques qui lui donnent corps. Le groupe est, d'autre part, toujours construit en relation avec d'autres : Gilles Laferté retrace les liens de sociabilité, les alliances matrimoniales, les conflits comme les mouvements de population au sein du Germanois et la manière dont cela participe à transformer - à stabiliser ou à faire imploser - les groupes qui s'y installent. L'approche est également attentive aux rapports de production et au travail : c'est bien dans la sphère productive que l'embourgeoisement s'ancre au départ. Cette dimension matérielle, qui occupe la première partie (et les quatre premiers chapitres) de l'ouvrage, se prolonge dans la seconde partie (et les trois derniers chapitres) autour de l'étude du style de vie des céréalier·es du Germanois. Celle-ci permet de comprendre pleinement l'un des arguments mis en lumière par l'auteur: l'enrichissement et l'embourgeoisement sont deux processus qui peuvent, certes, aller de pair, mais qui se déploient, non sans tension, dans des temporalités différentes.

\footnotetext{
${ }^{1}$ Cette démarche a été impulsée par un petit groupe de chercheurs travaillant à l'Institut national de la recherche agronomique (INRA), qui ont engagé un travail à la fois d'archivage de données déjà collectées et de croisement entre leurs enquêtes, lesquelles portaient sur un même espace. Ce travail a notamment donné lieu à un ouvrage collectif : Ivan Bruneau, Gilles Laferté, Julian Mishi et Nicolas Renahy (dir.), Mondes ruraux, classes sociales, Paris, Éd. de l'École des hautes études en sciences sociales, 2018.

${ }^{2}$ Gilles Laferté, « Des études rurales à l'analyse des espaces sociaux localisés », Sociologie, vol. 5, no 4, 2014, p. 424.

${ }^{3}$ L'approche relationnelle se retrouve dans de nombreux autres travaux de l'auteur, à l'instar de son travail sur les relations de crédit (Gilles Laferté, « De l'interconnaissance sociale à l'identification économique : vers une histoire et une sociologie comparées de la transaction à crédit », Genèses, $n^{\circ} 79,2010$, p. 135-149), sur l'identification économique (id., «L'identification économique », Genèses, n' 79, 2010, p. 2-5) et, plus largement, des réflexions qu'il a ouvertes sur les liens entre appartenance, identification et image sociale (Martina Avanza et Gilles Laferté, « Dépasser la "construction des identités" ? Identification, image sociale, appartenance », Genèses, n 61, 2005, p. 134-152).
} 
Comme le rappelle l'introduction, ce sont ainsi trois histoires que l'on peut lire en ouvrant cet ouvrage : l'histoire d'un espace rural - le Germanois - qui se transforme ; la trajectoire collective d'un groupe d'exploitant·es agricoles ; enfin celle d'un processus, l'embourgeoisement.

\section{Une entrée par l'espace}

Ce sont d'abord les atouts d'une entrée par l'espace local que le livre donne à voir. C'est en gardant la focale sur le Germanois qu'il est possible de construire une approche attentive aux relations nouées entre différents groupes sociaux dans le temps. Cette entrée permet notamment de comprendre la formation d'un groupe d'agriculteur·rices, sa stabilisation, son enrichissement et sa « dépaysannisation ${ }^{4}$ » progressive. Là où il est courant de s'arrêter à l'idée d'un territoire rural qui se vide, l'auteur s'attache à décrire minutieusement les transformations de cet espace polyvalent - l'histoire du Germanois est autant agricole qu'industrielle. En maintenant la focale sur cet espace, Gilles Laferté peut ainsi montrer combien l'enrichissement lent de celles et ceux qui s'y installent s'ancre dans la disponibilité du foncier qui fait suite aux nombreux départs (des notables notamment) advenus dans la seconde moitié du XIX ${ }^{\mathrm{e}}$ siècle. Le brassage migratoire important du début $\mathrm{du} \mathrm{XX}$ siècle et le jeu des alliances matrimoniales sédentarisent et clôturent progressivement un groupe d'agriculteur·rices, qui pratiquent alors autant l'élevage que la polyculture et partagent des moments de sociabilité avec leurs voisin $\cdot e \cdot s$ ouvrier $\cdot e \cdot s$.

Ce groupe s'épanouit au cours du siècle, moins par le nombre que par l'expansion foncière des exploitations, qui accompagne le mouvement d'abandon du lait et de l'élevage au profit de la production céréalière. L'approche localisée déployée dans l'ouvrage permet de mettre au jour trois formes d'inscription des céréalier·es dans le Germanois. D'abord, l'encastrement fort des relations marchandes dans les rapports d'interconnaissance façonne un marché foncier marqué par l'importance du capital d'autochtonie ${ }^{5}$. Les agriculteur·rices sont ensuite surreprésenté·es parmi les élu·es municipaux, l'ancrage dans une lignée familiale étant là aussi une ressource. Enfin, les céréalier·es du Germanois participent à la fabrique des paysages «par le travail des champs mais aussi par la construction et l'entretien du bâti » (p. 194). L'accroissement du capital économique se traduit en priorité par l'augmentation du patrimoine immobilier, que celui-ci soit professionnel - nouveaux bâtiments agricoles - ou privé, avec l'émergence et la multiplication des pavillons, mais aussi par la réhabilitation de vieilles bâtisses ou, plus rarement, d'anciennes demeures de notables ${ }^{6}$.

Si l'espace enregistre autant qu'il participe à l'épanouissement des céréalier·es du Germanois, il enferme et contraint également la mobilité du groupe. Le pouvoir sur le foncier bute ainsi rapidement sur la montée des prix à mesure que la terre se fait rare. Il se trouve aussi limité par les structures syndicales et les jeux de réputation locale, lorsqu'une famille, trop gourmande, commence à accumuler les terres dans des proportions que le voisinage juge trop importantes. L'accès au pouvoir politique se heurte toujours au faible accès des agriculteurs élus localement à d'autres arènes politiques : l'ancrage local peut être une ressource au niveau communal, mais pèse moins par rapport à d'autres formes de légitimité sur les scènes politiques départementale, régionale ou nationale. Quant aux paysages, une tension se fait jour dans l'ouvrage autour des nombreuses acquisitions immobilières. Celles-ci sont des marqueurs ostensibles de l'enrichissement, mais « révèlent un goût populaire en grand », plutôt qu'elles ne signent l'adoption d'un goût ou de stratégies immobilières bourgeoises. L'ennoblissement du pavillon est ainsi préféré à la réhabilitation de l'ancien ; l'immobilier est rarement conçu comme un

\footnotetext{
${ }^{4}$ C'est le terme employé par l'auteur pour décrire le mouvement d'éloignement d'avec le mode de vie paysan.

${ }^{5}$ Jean-Noël Retière, « Autour de l'autochtonie. Réflexions sur la notion de capital social populaire », Politix, $\mathrm{n}^{\circ}$ 63, 2003, p. 121-143.

${ }^{6}$ Les résidences des agriculteurs comme leurs acquisitions immobilières sont minutieusement décrites dans l'ouvrage, à l'appui de photographies notamment, en particulier dans le chapitre 7.
} 
patrimoine de jouissance et est acquis dans un espace circonscrit; en somme, il ne marque pas l'entrée dans un mode de vie affranchi de cet espace localisé.

On relève au total au moins trois atouts d'une entrée par l'espace local : la sortie d'une représentation fixe des campagnes, la mise au jour de la mobilité d'un groupe par son ancrage dans l'espace, enfin l'attention portée aux traductions spatiales et locales de cette mobilité. L'approche localisée de la structure sociale rappelle surtout l'absence de structure sociale unidimensionnelle. Comme l'affirme l'auteur dès l'introduction, une des ambitions de l'ouvrage est de montrer que «les mobilités sociales concrètes ne sont pas des déplacements neutres dans une structure sociale linéaire verticale » (p. 17). La question de l'articulation entre structure nationale et locale reste pour autant particulièrement difficile à penser. L'ouvrage rend pleinement compte, dans cet espace local, de la domination et de l'éloignement progressifs des céréalier-es sur et vis-à-vis des classes populaires en particulier. Mais l'argument qui suit le chemin inverse, celui de la subordination des agriculteur rices du Germanois en regard de la culture légitime, est plus fragile. Renvoyant à un débat ancien $^{7}$, cet argument repose sur le postulat qu'il existe une échelle des goûts. Si Gilles Laferté note que « les styles de vie peuvent difficilement se catégoriser dans une classification unidimensionnelle» (p. 262), il semble hésiter à sauter complètement le $\operatorname{pas}^{8}$. Cette légère hésitation est, en un sens, assumée par l'auteur, qui remarque ainsi après la description de la demeure d'un des enquêtés, que cette dernière, «vue du capital culturel légitime », incarne «le mauvais goût du parvenu » et « frise le ridicule », mais qu'elle « plaît aux entrepreneurs locaux » et, d'après son propriétaire, « fait même des jaloux » (p. 304-305). C'est ainsi autour de l'articulation entre scène locale et nationale que se noue une énigme : comment s'actualise empiriquement la subordination culturelle des céréalier es si, localement, il n'en est rien ? L'approche localisée des groupes sociaux ne fait-elle pas en partie voler en éclat l'idée de structure sociale nationale si, comme le souligne l'auteur, cette dernière « se rediffracte constamment par les logiques toujours spatialisées du social » (p. 45) ? En somme, peut-on vraiment articuler approche par les espaces sociaux localisés et approche localisée de la structure sociale ?

\section{La trajectoire collective d'un groupe d'agriculteur ·rices}

Le livre suit un deuxième fil directeur autour de la catégorie sociale des agriculteur-rices. Il s'agit d'apporter une nouvelle pierre à la sociologie de ce groupe, notamment pour « rééquilibrer l'image construite de l'agriculture et des agriculteurs » (p. 34), laquelle est souvent misérabiliste. En prenant pour objet les générations de céréalier es du Germanois - soit des agriculteur rices à la tête de grandes exploitations, doté es de revenus, comme d'un patrimoine les situant nettement parmi les plus fortunés de ce groupe ${ }^{9}-$, l'auteur donne à voir la diversité de la catégorie « agriculteur·rices », perceptible même au sein des céréalier·es du Germanois, et interroge aussi en retour la portée de cette dernière.

Le grand mouvement que dessine l'ouvrage est celui d'une "dépaysannisation » progressive des céréalier·es du Germanois. Elle s'incarne de trois manières. La première tient au contenu du travail. L'arrêt de l'élevage et de la production de lait, la mécanisation croissante des activités agricoles après-guerre comme la taille importante des exploitations réduisent la part des tâches les plus manuelles au profit d'activités managériales et techniciennes. Lea paysan·ne, dont

\footnotetext{
${ }^{7}$ Voir notamment l'ouvrage de Claude Grignon et Jean-Claude Passeron, Le savant et le populaire. Misérabilisme et populisme en sociologie et en littérature, Paris, Seuil, 1989.

${ }^{8}$ Comme le note l'auteur, cette hésitation renvoie aussi à une difficulté méthodologique : « Il est tout de même saisissant de voir combien l'activité de classement social par la mise en scène de l'habitat (et l'on pourrait dire la même chose de l'apparence vestimentaire) structure, comme un réflexe, l'ensemble des interactions sociales de nos enquêtés, formant la matière première de leur sociologie ordinaire, mais aussi de constater la difficulté des sciences sociales à s'emparer de front de ces objets » (p. 262).

$992 \%$ des agriculteurs du Germanois peuvent être classés dans la catégorie « gros exploitants » de l'enquête Patrimoine (INSEE, 2010). La médiane du patrimoine de ces agriculteurs (779 $470 €$ ) est plus importante que celle des cadres et bien plus élevée que celle des agriculteurs dans leur ensemble (p. 181).
} 
l'auteur rappelle la proximité avec l'ouvrier·e par les tâches effectuées, est dès lors plus proche de la figure patronnale. Cette transformation du contenu des tâches se décline au niveau de l'emploi du temps, désormais moins contraint, ce dont témoigne l'augmentation des départs en vacances. La deuxième dimension, peu séparable de la première, est la disjonction entre les collectifs de travail et les familles agricoles. L'auteur souligne combien le modèle familial traditionnel paysan, lequel implique l'ensemble de la maisonnée autour de l'exploitation, facilite au départ la croissance capitalistique des entreprises familiales. Mais il montre qu'en retour, l'enrichissement rend possible l'individualisation, la «déconjugalisation des formes agricoles contemporaines »- les conjoint·es d'agriculteur·rices travaillent de plus en plus souvent dans d'autres métiers - et, plus largement, la séparation entre sphères privée et professionnelle. Cette disjonction se lit notamment dans le mouvement d'empavillonnement des agriculteur rices, qui acte l'éloignement entre résidence principale et exploitation. Elle se prolonge dans la transformation du modèle d'entreprise adossée aux exploitations, par l'adoption rapide et généralisée de la forme sociétaire et la financiarisation croissante du capital, laquelle est plus endogène (par l'investissement régulier) qu'exogène. Enfin, la dernière dimension tient au rapport à l'école. Gilles Laferté souligne que c'est hors de l'école et autour d'une « stratégie agricole » - installer sur de nouvelles exploitations la descendance que la reproduction du groupe est assurée dans les années 1960. Mais, là aussi, il y a dépaysannisation, par la conversion progressive à l'école et aux diplômes ${ }^{10}$. La génération suivante est ainsi plus diplômée. La reprise de l'exploitation, loin d'être la norme, s'en trouve alors de plus en plus coûteuse et dévaluée par rapport aux carrières, hors agriculture, qu'ouvre la détention de diplômes du supérieur.

La dépaysannisation, qui est aussi un mouvement de rapprochement des céréalier ·es du Germanois du groupe des cadres et des petit·es patron·nes, interroge in fine la cohérence et le caractère heuristique de la catégorie « agriculteur·rices ». Au terme de la lecture, on se demande si le livre acte une fonte ou une refonte de ce groupe. Pour répondre à cette question, une des pistes aurait pu consister à interroger davantage les différents collectifs référents des céréalier ·es. Si l'auteur montre bien que ces dernier'es ne se considèrent jamais comme des « bourgeois·es », on sait finalement peu s'ils se définissent toujours comme des agriculteur rices, et si, au-delà de l'affiliation par le métier, cette catégorie est encore le support de logiques d'appartenance. On pourrait, par exemple, étudier ces logiques d'appartenance autour de la représentation politique des intérêts des agriculteur·rices - notamment par l'examen de la place et l'investissement des céréalier·es dans les différents syndicats agricoles - que l'ouvrage laisse davantage dans l'ombre.

\section{Une histoire d'embourgeoisement}

Nous distinguons ici le mouvement de «dépaysannisation » que dessine l'ouvrage de celui qui lui donne son titre - l'embourgeoisement. Un des apports importants du travail de Gilles Laferté consiste en effet dans son effort de conceptualisation de dynamiques - gentrification, embourgeoisement, dépaysannisation, enrichissement - qui, souvent, sont présentées soient comme allant mécaniquement de pair, soit comme interchangeables. S'il propose une entrée par l'espace localisé et s'il constitue une contribution importante à la sociologie des agriculteur·rices, l'ouvrage est peut-être d'abord un travail qui renouvelle l'appréhension des mobilités sociales.

Avant de présenter les principaux résultats, revenons sur les atouts de l'approche proposée. C'est le plus souvent le changement de catégorie socioprofessionnelle qui est utilisée pour décrire les mobilités sociales. En s'attachant à suivre la trajectoire collective d'un groupe à l'intérieur d'une même catégorie socioprofessionnelle, l'ouvrage redonne de l'épaisseur

\footnotetext{
${ }^{10}$ Cette transformation est en grande partie structurelle, mais l'auteur note que les agriculteurs « ont bénéficié plus que les autres catégories sociales de la démocratisation scolaire » (p. 229).
} 
à une forme de mobilité - la mobilité structurelle - qui a peu été investiguée ${ }^{11}$. L'analyse des trajectoires sociales des céréalier·es du Germanois s'inscrit également dans la lignée du programme de recherche, développé notamment dans le dossier sur les mobilités paru dans la revue Politix ${ }^{12}$, autour des mobilités qui ne se déploient ni par le diplôme, ni par l'accumulation de capital culturel, mais qui passent d'abord par l'accumulation de capital économique.

Un des résultats importants du travail de Gilles Laferté consiste à démontrer qu'enrichissement et embourgeoisement ne sont pas similaires. L'accumulation de capital économique n'est pas, en soi, un embourgeoisement : « une catégorie sociale ne s'adapte pas spontanément à la bourgeoisie à mesure de l'augmentation de ses revenus » (p. 21). Celui-ci accompagne bien souvent cette accumulation, mais dans une temporalité bien plus longue, que le suivi de différentes générations de céréalier es du Germanois permet de saisir. Par embourgeoisement, Gilles Laferté désigne ainsi moins l'augmentation de capital économique en elle-même que le fait que celle-ci soit «guidée par la reconnaissance de la légitimité de l'ordre social existant et du mode de vie des dominants » (p. 261). La transformation du rapport à l'école, évoquée précédemment, renvoie pleinement à ce type de dynamique. À partir de son analyse des intérieurs, Gilles Laferté expose également le caractère mimétique, et maladroit, du goût des céréalier-es. C'est sur ce point qu'il propose une distinction avec la gentrification comme forme de mobilité qui passe par la critique et la subversion de la culture légitime et par le renouvellement des formes de distinction ${ }^{13}$. L'embourgeoisement désigne plutôt les situations dans lesquelles l'accumulation de capital se double d'efforts pour «se mettre à l'épreuve des catégories supérieures » (p. 359). L'embourgeoisement des céréalier·es rend compte in fine de l'émergence d'une nouvelle fraction des classes supérieures, qui participe à la reconstitution du pôle économique de ces dernières. Cet effort de conceptualisation offre la possibilité de penser les trajectoires collectives d'autres groupes sociaux, en opérant des distinctions qui leur donnent davantage d'épaisseur que lorsqu'il s'agit simplement de constater un changement de catégorie socioprofessionnelle.

La dimension processuelle de l'embourgeoisement, sur laquelle insiste l'auteur, est directement contenue dans le terme. Comme pour toutes les notions qui portent l'idée d'un changement - gentrification, financiarisation, urbanisation, etc. -, le risque est grand de se cacher derrière le mot sans que l'on parvienne à comprendre les ressorts et les inflexions du processus dévoilé. Dans l'ouvrage, cette dimension processuelle apparaît moins par le recueil de petits changements qui dessineraient un mouvement plus grand que par l'examen de toutes les tensions et résistances qui accompagnent l'enrichissement. Cela permet directement de renforcer l'argument de l'auteur quant à la disjonction relative entre enrichissement et embourgeoisement, tout en montrant, en creux, l'inscription dans un temps long de l'ensemble des changements dans le style de vie et au travail, qui lui permettent de parler d'embourgeoisement.

Une série de tensions voit le jour à mesure de l'enrichissement. Ainsi, l'« ethos du faire » des agriculteur rices, leur ascétisme et la place importante donnée au travail, sont à la fois bien ajustés à l'esprit entrepreneurial et favorisent ainsi l'accumulation du capital économique, mais constituent un frein à l'entrée dans un mode de vie bourgeois, marqué par l'oisiveté, la pratique de certains loisirs, la consommation distinctive ou la délégation de certaines tâches. C'est d'ailleurs seulement dans les générations les plus récentes que l'auteur observe la pratique fréquente de loisirs bourgeois, comme le ski, le golf ou la natation. De même, si l'accumulation économique se traduit au départ par la constitution d'un patrimoine foncier et immobilier, le recours au placement financier est lent. Il rencontre la résistance d'un comportement prudentiel

\footnotetext{
${ }^{11}$ Elle l'est souvent au sein de travaux portant moins sur un groupe que donnant une vue d'ensemble (voir Louis-André Vallet, « Mobilité entre générations et fluidité sociale en France. Le rôle de l'éducation », Revue de l'OFCE, vol. 150, 2017, p. 27-67.

${ }^{12}$ Voir notamment Julie Pagis et Paul Pasquali, « Observer les mobilités en train de se faire. Micro-contextes, expériences vécues et incidences socio-politiques », Politix, $\mathrm{n}^{\circ}$ 114, 2016, p. 7-21.

${ }_{13}$ Voir Anaïs Collet, Rester bourgeois. Les quartiers populaires, nouveaux chantiers de la distinction, Paris, La Découverte, 2015.
} 
et averse au risque, qui tient à l'aléa très fort qui entoure l'activité principale des agriculteur rices. La financiarisation est finalement moins la résultante mécanique de l'accumulation que le produit du travail des conseiller·es bancaires du Crédit agricole, ce qui rappelle que l'embourgeoisement n'est jamais solitaire et n'est intelligible qu'en considérant les interactions nouées entre différents groupes - les agriculteur rices et leurs conseiller·es bancaires notamment. Une autre tension tient au rôle pilier de l'État, qui, au travers des subventions européennes, participe à stabiliser et lisser les revenus des céréalier·es du Germanois, et partant à les enrichir, tout en les plaçant dans une position qui rend difficile la tenue d'une rhétorique méritocratique de légitimation de la fortune. Un dernier exemple de tension réside dans le rapport à l'école. Comme nous l'avons souligné, les familles agricoles ont fait entrer les diplômes dans leurs modes de reproduction. Mais Gilles Laferté note qu'elles ont gardé un rapport singulier et ambivalent à l'école : toutes « ne jouent pas complètement le jeu de l'ouverture » et elles sont nombreuses à spécialiser l'orientation de leurs enfants dans les filières techniques (p. 236). Si le passage par l'école s'impose désormais, l'horizon reste celui de la préservation d'un outil de production, dont le destin scolaire ne doit pas trop éloigner. La mise au jour de ces tensions donne à voir le processus long de l'embourgeoisement et l'absence de lien simple avec l'enrichissement. Le recours à un terme qui désigne en lui-même un processus butte toutefois toujours sur la question de la fin: si, comme le souligne l'auteur, les céréalier-es ne peuvent devenir des bourgeois·es, où s'arrête l'embourgeoisement? La réponse de l'ouvrage serait de dire qu'il ne s'arrête pas, les agriculteur-rices étant «irrémédiablement en infériorité sociale face aux plus certifiés culturellement» (p. 360). Mais si l'on suit dans le même temps l'idée de reconstitution d'un pôle économique au sein des classes supérieures et si l'on s'arrête sur le contexte de revalorisation du capital économique ${ }^{14}$ et de célébration de la figure de l'entrepreneur ${ }^{15}$, nous pouvons également penser que l'infériorité sociale des embourgeoisé·es n'est pas une donnée stabilisée et peut également être envisagée dans une perspective dynamique.

L'ouvrage de Gilles Laferté, en proposant de contribuer à une sociologie des agriculteur rices, ouvre de nombreuses pistes pour appréhender les mobilités sociales, pour penser l'articulation entre appartenance sociale et enrichissement, enfin pour dresser un tableau du monde rural attentif aux multiples connexions et dynamiques qui le façonnent. Loin d'une perspective figée et clôturée des campagnes, l'ouvrage offre une analyse importante des luttes des classes et de classements qui se jouent en leur cœur, mais aussi au sein des classes supérieures.

\footnotetext{
${ }^{14}$ Étienne Gérard et Anne-Catherine Wagner, «Introduction : Élites au Nord, élites au Sud : des savoirs en concurrence ? », Cahiers de la recherche sur l'éducation et les savoirs, ${ }^{\circ}$ 14, 2015, p. 7-24.

${ }^{15}$ Sarah Abdelnour, Moi, petite entreprise. Les autoentrepreneurs, de l'utopie à la réalité, Paris, Puf, 2017.
} 